\title{
Fourier Analysis of Ronchigram and Aberration Assessment
}

\author{
K. Ishizuka, ${ }^{* * *}$ K. Kimoto, ${ }^{* *}$ Y. Bando** \\ * HREM Research Inc. 14-48 Matsukazedai, Higashimatsuyama, 355-0055 Japan \\ ** National Institute for Materials Science, 1-1 Namiki, Tsukuba, 305-0044, Japan.
}

A shadow image obtained from amorphous/non-periodic materials has been used for a manual alignment of a scanning transmission electron microscope (STEM), and proposed to measure aberrations quantitatively [1]. A Ronchigram, a shadow image obtained from crystal materials in STEM, can also be used to check an alignment quality [2-4]. It was shown that a Fourier transform of a Ronchigram might be used to measure aberrations [5-7]. This report revisits Fourier analysis of a Ronchigram to give a more concrete mathematical foundation.

We will confine here to a weak phase object and ignore interference between scattered waves. Thus, it will be enough if we consider an interference term between the center beam and $+\mathrm{g}$ reflection, and amplitude of the two-beam Ronchigram may be given

$$
\Phi\left(\vec{k}, \vec{r}_{o}\right)=\exp \left(2 \pi i \vec{k}_{r_{o}}\right)\left[A(\vec{k}) \exp \left\{\frac{2 \pi}{\lambda} i \chi(\vec{k})\right\}+i Q(\vec{g}) \exp \left(-2 \pi i \vec{g} \vec{r}_{o}\right) A(\vec{k}-\vec{g}) \exp \left\{\frac{2 \pi}{\lambda} i \chi(\vec{k}-\vec{g})\right\},\right.
$$

where $r_{o}$ is a probe position, $\chi(\vec{k})$ a wave aberration function and $Q(\vec{g})$ a structure factor for a reflection $\mathrm{g}$. By neglecting a second order term an observed Ronchigram (intensity) is given

$$
R\left(\vec{k}, \vec{r}_{o}\right)=\Phi\left(\vec{k}, \vec{r}_{o}\right) \cdot \Phi^{*}\left(\vec{k}, \vec{r}_{o}\right) \approx A(\vec{k})+i f_{g}\left(\vec{k}-\frac{1}{2} \vec{g}, \vec{r}_{o}\right)-i f_{g}^{*}\left(\vec{k}-\frac{1}{2} \vec{g}, \vec{r}_{o}\right),
$$

where

$$
f_{g}\left(\vec{k}, \vec{r}_{o}\right) \equiv A\left(\vec{k}+\frac{1}{2} \vec{g}\right) A\left(\vec{k}-\frac{1}{2} \vec{g}\right) Q(\vec{g}) \exp \left(-2 \pi i \vec{g} \vec{r}_{o}\right) \exp \left\{\frac{2 \pi i}{\lambda}\left[\chi\left(\vec{k}-\frac{1}{2} \vec{g}\right)-\chi\left(\vec{k}+\frac{1}{2} \vec{g}\right)\right]\right\} .
$$

Then, we will get a Fourier transform of a two-beam Ronchigram as

$$
\Psi\left(\vec{h}, \vec{r}_{o}\right) \equiv F T\left[R\left(\vec{k}, \vec{r}_{o}\right)\right]=a\left(\vec{h}, \vec{r}_{o}\right)+i\left[F_{g}\left(\vec{h}_{,} \vec{r}_{o}\right)-F_{g}^{*}\left(-\vec{h}, \vec{r}_{o}\right)\right] \exp (\pi i \vec{g} \vec{h}),
$$

where $a\left(\vec{h}, \vec{r}_{o}\right)$ is an Airy disk, and $F_{g}\left(\vec{h}, \vec{r}_{o}\right)$ is a Fourier transform of $f_{g}\left(\vec{k}, \vec{r}_{o}\right)$. Figure 1 shows a two-beam Ronchigram and its Fourier transform, where you can see a pair of comet-shaped spot due to spherical aberration. Each comet comes from $F_{g}\left(\vec{h}, \vec{r}_{o}\right)$. It was shown that the position from the origin changes with defocus and two-fold astigmatism, but the shape remains constant and the angle of the comet tail that is always $60^{\circ}$ [5]. However, the reason of the comet shape remains unresolved.

When we consider a symmetric three-beam case, namely the center beam and $+\mathrm{g}$ and $-\mathrm{g}$ reflections, using Friedel's law for $Q(\vec{g})$ the Ronchigram may be written as

$R\left(\vec{k}, \vec{r}_{o}\right)=\Phi\left(\vec{k}, \vec{r}_{o}\right) \cdot \Phi^{*}\left(\vec{k}, \vec{r}_{o}\right) \approx A(\vec{k})+i f_{g}\left(\vec{k}-\frac{1}{2} \vec{g}, \vec{r}_{o}\right)-i f_{g}^{*}\left(\vec{k}-\frac{1}{2} \vec{g}, \vec{r}_{o}\right)+i f_{g}^{*}\left(\vec{k}+\frac{1}{2} \vec{g}, \vec{r}_{o}\right)-i f_{g}\left(\vec{k}+\frac{1}{2} \vec{g}, \vec{r}_{o}\right)(5)$

and its Fourier transform for the three-beam Ronchigram results:

$$
\Psi\left(\vec{h}, \vec{r}_{o}\right)=a\left(\vec{h}, \vec{r}_{o}\right)+i\left[F_{g}\left(\vec{h}, \vec{r}_{o}\right)-F_{g}^{*}\left(-\vec{h}, \vec{r}_{o}\right)\right]\{\exp (\pi i \vec{g} \vec{h})-\exp (-\pi i \vec{g} \vec{h})\} .
$$

Thus, the Fourier transform of a symmetric three-beam Ronchigram shows fine straight fringes perpendicular to the scattering vector, $\boldsymbol{g}$, due to the last terms in the curly brackets. The origin of these fringes was attributed to the presence of two identical sets of fringes in the Ronchigram [5]. 
The presence of two identical patterns shifted by $\pm \frac{1}{2} \vec{g}$ is mathematically shown in Eq. (5).

The Fourier transform $F_{g}\left(\vec{h}, \vec{r}_{o}\right)$ may be given by a far field distribution of $f_{g}\left(\vec{k}, \vec{r}_{o}\right)$. Then, the comet shape will be determined by a gradient of a wave front controlled by the phase term. We may note that the total spot displacement may be estimated as a sum of gradients of constituent aberrations. Thus, the shift of a spot position due to spherical aberration will be given by

$$
\nabla \chi_{40}\left(\vec{k}-\frac{1}{2} \vec{g}\right)-\nabla \chi_{40}\left(\vec{k}+\frac{1}{2} \vec{g}\right)=-2 c_{40}(\vec{k} \vec{g}) \vec{k}-c_{40}\left(k^{2}+\frac{1}{4} g^{2}\right) \vec{g} \quad \text {. }
$$

where $c_{40}=C_{s} \pi \lambda^{3} / 2$. Now, to simplify the argument we will define the new axis of coordinates, where the $\mathrm{x}$-axis aligns the scattering vector $\boldsymbol{g}$, and the origin is placed at the comet head, namely $\left(c_{40}\left(\frac{1}{2} g_{x}\right)^{2} g_{x}, 0\right)$. Then, the $\mathrm{x}$ and $\mathrm{y}$ components of the above gradient are respectively given by

$$
X=c_{40} g_{x}\left(3 k_{x}^{2}+k_{y}^{2}\right) ; Y=c_{40} g_{x}\left(2 k_{x} k_{y}\right) \text {. }
$$

The position of the comet tail corresponds to the caustic determined by these coordinates. In order to verify this statement, we consider the angle $\alpha$ corresponding to $(X, Y)$ using the polar coordinates for $\vec{k}$, namely $k_{x}=r \cos \theta, k_{y}=r \sin \theta$,

$$
R=\tan \alpha=\frac{Y}{X}=\frac{\sin 2 \theta}{2 \cos ^{2} \theta+1}
$$

It is easily shown that $R$ has an extremum at $\theta= \pm 60^{\circ}$, and then $\alpha=30^{\circ}$. Thus, the comet angle is $60^{\circ}$ as observed by Boothryod [5]

The same argument on the shift of a spot position due to defocus and two-fold astigmatism gives the identical results derived from $\chi(\vec{k}-\vec{g})-\chi(\vec{k})$ [5-7]. However, it becomes clear from the argument for spherical aberration that the phase term $\chi\left(\vec{k}-\frac{1}{2} \vec{g}\right)-\chi\left(\vec{k}+\frac{1}{2} \vec{g}\right)$ in Eq. (3) is more fundamental for Fourier analysis of the Ronchigram.
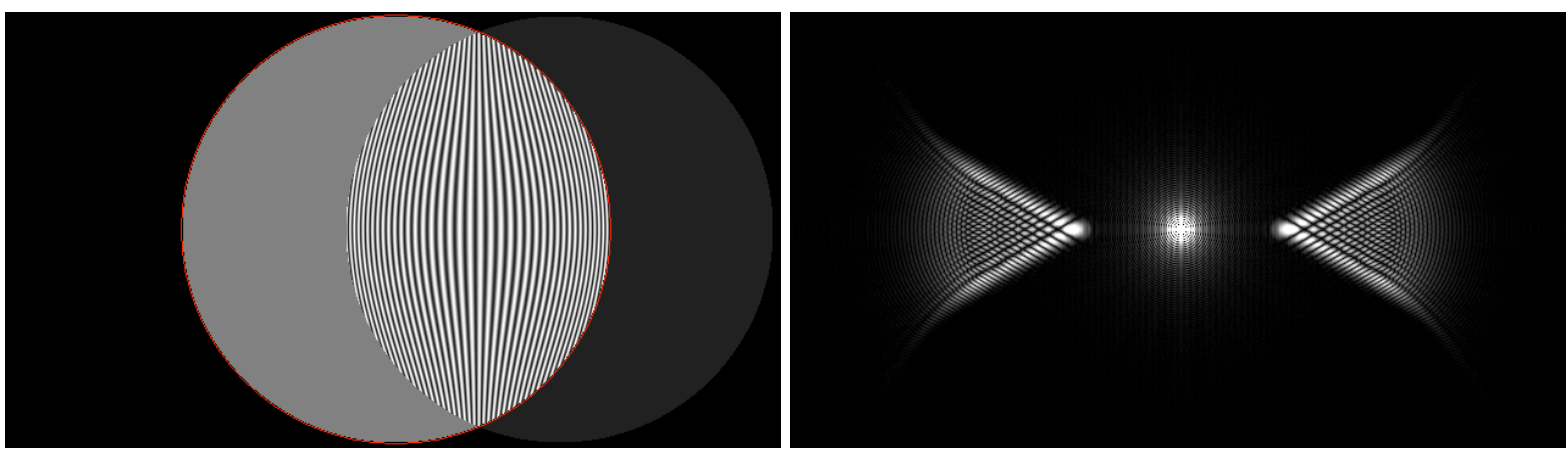

Figure 1. Two-beam Ronchigram (left) and its Fourier transform. This is a kinematical simulation and wave aberration is considered. $100 \mathrm{kV}, \mathrm{Cs}=3.1 \mathrm{~mm}$, defocus $=350 \mathrm{~nm}, \mathrm{~g}=3.07 \mathrm{~nm}^{-1}$.

[1] N. Dellby et al., J. Electron Microsc. 50 (2001) 177-185.

[2] J. A. Lin and J. M. Cowley, Ultramicroscopy 19 (1986) 31-42.

[3] N. D. Browning et al., J. Electron Microsc. 50 (2001) 205-218.

[4] Q. M. Ramasse and A. L. Bleloch, Ultramicroscopy, 106 (2005) 37-56.

[5] C. B. Boothroyd, Scanning Microscopy, 11 (1997) 31-42.

[6] A. R. Lupini and S. J. Pennycook, J. Electron Microsc. 57 (2008) 195-201.

[7] K. Kuramochi et al., Ultramicroscopy, 108 (2008) 339-345. 